\title{
Potential Effect of Cyclophosphamide on Bleb Survival in Five Patients with Multiple Sclerosis Who Underwent Glaucoma Surgery
}

\author{
Andrea Giudiceandrea - Maria Emanuela Toro (D) - Andrea Scupola • \\ Aldo Caporossi · Viviana Nociti · Massimiliano Mirabella • \\ Tommaso Salgarello
}

Received: April 7, 2018 / Published online: June 7, 2018

(C) The Author(s) 2018

\section{ABSTRACT}

Introduction: The purpose of this case series was to report the potential role of cyclophosphamide (CY) on bleb survival and to evaluate the safety of the trabeculectomy procedure under immunosuppressant systemic therapy.

Case series: Five eyes of five patients with unresponsive to intraocular pressure (IOP) lowering medication, progressive glaucoma, underwent mytomicin C (MMC) augmented phaco-trabeculectomy, performed by the same surgeon, A.G., during the period from May 2015 to January 2016. All patients were treated with low doses of systemic CY at the time of surgery, to control their relapsing progressive multiple sclerosis (MS) form.

Enhanced digital features To view enhanced digital features for this article go to https://doi.org/10.6084/ m9.figshare.6383123.

A. Giudiceandrea · M. E. Toro $(\bowtie) \cdot$ A. Scupola .

A. Caporossi · T. Salgarello

Ophthalmology Division - Department of Head and

Neck Surgery of Catholic University of "Sacro

Cuore", Fondazione Policlinico Universitario

"A. Gemelli", Rome, Italy

e-mail: emanuelatoro@tiscali.it

V. Nociti · M. Mirabella

Catholic University of "Sacro Cuore", Fondazione

Policlinico Universitario “A. Gemelli", Rome, Italy

V. Nociti

Don C. Gnocchi Foundation Onlus, Milan, Italy
Results: During a mean follow-up period of $20.6 \pm 8.1$ months, for cases of "complete success" (when the IOP was $<15 \mathrm{mmHg}$ without glaucoma therapy) were observed, while one case was classified as a "qualified success" since the IOP was $\leq 15 \mathrm{mmHg}$ with $\beta$-blocker drops. There were no bleb infections, nor bleb-related complications.

Conclusion: This study reports the safety of performing the filtration surgical procedure under immunosuppressant systemic therapy and provides a possible explication of $\mathrm{CY}$ antifibrotic mechanism and its possible role on bleb survival. Our findings may suggest new perspectives of study in this field.

Keywords: Anti-fibrotic; Bleb survival; Cyclophosphamide; Immunosuppressants; Multiple sclerosis; Trabeculectomy

\section{INTRODUCTION}

Based on evidence that IOP lowering is the exclusive therapy of open angle glaucoma [1], when IOP cannot be adequately controlled with medical therapy or currently available laser procedures, the management options to prevent visual impairment and blindness require surgical approaches; currently, despite its downward trend, trabeculectomy (i.e. anterior, penetrating, ab externo filtering procedure) is the standard glaucoma filtration surgery in the 
world $[1,2]$. Scleral bleb results from fluid accumulation, meaning procedure success. After filtration surgery, vascularity increase and fibrotic reaction on bleb site are considered to be a physiological response against tissue injury; consequently, in a few hours to the first 1-2 weeks after surgery [3], changes in epithelial and mesenchymal cells of conjunctiva occurs, i.e. the Tenon fibroblast activation and proliferation at the site of the fistula induces a inflammation, formation of granulation tissue, collagen deposition and extracellular matrix (ECM) remodeling [4]. This process impairs filtering bleb formation and the aqueous humor outflow facility $[4,5]$. Treatment with cytostatics has been established to reduce the proliferation of connective tissue around the bleb. Actually MMC represents the most efficacious anti-fibrotic agent used as intraoperative strategy to attenuate fibrosis after trabeculectomy and to prolong the bleb survival [6].

MS is a chronic inflammatory and neurodegenerative demyelinating disease of the central nervous system (CNS) [7]. Immunosuppressive drugs (azathioprine, CY, mitoxantrone, etc.) have been employed successfully in patients with autoimmune disease and, therefore, in MS also. They are currently not formally approved for MS (except for mitoxantrone) and thus are used off-label. Nonetheless, immunosuppressive drugs continue to be used in MS patients, particularly in some patients refractory to conventional therapies, or in chronic progressive form that requires immunosuppression because of rapid progression of disability. $\mathrm{CY}$ is an alkylating agent able to interfere with DNA integrity and function, commonly used as a cytotoxic medication for cancer chemotherapy or to prevent rejection after organ transplantation $[8,9]$. Several studies reported on the efficacy of CY in stabilizing patients with selected forms of MS; moreover, low doses of CY therapy represents a well-tolerated therapeutic course in MS patients [10, 11].

In spite of being a general immunosuppressant agent, CY shows quite selective immune effects in MS: some authors suggest that CY may pilot the immune balance from a Th1/Th17 immune stance, known to be deleterious in MS, towards $\mathrm{T}$ helper type $2\left(\mathrm{~T}_{\mathrm{H}} 2\right)[12,13]$ and
Th $3_{\text {REG }}$ responses [14]. Our work is mainly intended to demonstrate the absence of an increased risk of infection in patients with MS receiving $\mathrm{CY}$, but at the same time strongly suggests a possible protective role of such drug against the fibrotic complications often challenging glaucoma surgery success.

\section{METHODS}

We report the cases of five patients, one man and four women, with relapsing progressive MS. Data are reported without a prospective trial registration, due to the absence of a comparative, controlled clinical trial set-up. The mean age at the time of surgery was $57.16 \pm 7.31$ years. Informed consent was obtained from all individual patients for inclusion in the series. All patients presented with glaucoma with a progressive and severe visual field (VF) loss, defined as a VF mean deviation $(\mathrm{MD}) \leq-10 \mathrm{~dB}$ and a pattern standard deviation (PSD) $>6 \mathrm{~dB}$, despite maximally tolerated medications. IOP measurements were all obtained by Goldmann applanation tonometry. Visual field tests was conducted with a Humphrey Visual Field Analyzer I set for the central 32 threshold test standard, size III white stimulus, with the foveal threshold test turned on. One patient presented a high risk of procedure failure because of a previous failed phaco-trabeculectomy 1 year before the diagnosis of MS. All MS patients were treated with CY, $1 \mathrm{~g}$ i.v. monthly, for a total cumulative dose administered of $2.5 \mathrm{mg}$ to $3.5 \mathrm{mg} / \mathrm{mm}^{2}$. Following a mean of 6 i.v. CY treatments and a therapy-free month break, all patients were switched to oral immunosuppressive therapy of $50 \mathrm{mg}$, as a daily dose of maintenance.

The patients were actually taking $50 \mathrm{mg}$ /day CY orally from at least 2 months (range 2-3) when MMC-enhanced primary phaco-trabeculectomy was performed. One eye, with previous failed phaco-trabeculectomy, underwent a new ab externo filtering procedure augmented with MMC. In all cases a standardized filtration surgery procedure was performed. A fornix-based conjuntival flap was created, with gentle cauterization of the exposed sclera. The 
dissection of the $4 \times 4 \mathrm{~mm}$ scleral flap was followed by the application during $4 \mathrm{~min}$ of $0.2 \mathrm{mg} / \mathrm{mL}$ of MMC. After washing, 100 to 150 microns of corneal-scleral tissue was punched through via sclerotomy. Finally 10-0 nylon sutures were used for scleral flap approximation, and conjunctival closure. No intraoperative complication occurred. Removal of conjuntival sutures occurred at 20th-30th post-operative day in all patients. The IOP measurement was strictly done (every 3 days) in the post-operative 15 days, then monthly for up to 1 year; perimetry analysis was conducted every 3 months. Four patients kept the CY oral regimen throughout follow-up period. Case n.1 interrupted oral CY 3 months after surgery due to recurrent cystitis.

\section{RESULTS}

Based on the Advanced Glaucoma Intervention Study data [15] that recommends the aggressive IOP lowering with respect to the baseline in patients with advanced glaucoma and with appropriate life expectancy, the success of the surgical procedure in this group of patients was defined as a IOP $\leq 15 \mathrm{mmHg}$. The mean preoperative IOP of $20.2 \pm 1.6 \mathrm{mmHg}$ decreased to $13 \pm 2.6 \mathrm{mmHg}$ as registered at last follow-up (Table 1). In four patients the success was "complete" (the IOP was $<15 \mathrm{mmHg}$ without glaucoma therapy) and in one patient it was "qualified" because of beta-blockers additional treatment was needed (Table 1). At the 15th post-operative day the latter underwent to $0.02 \%$ MMC sub-Tenon's bleb needling because of bleb vascularity and increasing IOP (Table 1). There were no bleb infections, nor bleb related complications (Fig. 1); none of the patients experienced a VF defect relevant change over the entire follow-up period $20.6 \pm 8.1$ months nor developed severe adverse events due to oral CY.

\section{CONCLUSION}

In our surprising study experience, the satisfactory clinical course after the surgeries in patients with the same underlying condition and systemic CY protocol focused our attention on the possible role of CY's fibrosis cascade modulation. The analysis of post-operative clinical course in 39 trabeculectomies performed by the surgeon (A.G.) in the last 2 years in non MS-patients, reveals a vascularized bleb incidence at the 15th post-operative day in 63\% of patients with an mean IOP of $21 \mathrm{mmHg}$ without glaucoma therapy. Many conditions have been regarded as risk factors for bleb failure (ethnicity, younger age, ocular inflammation, neovascular glaucoma, duration and number of topical anti-glaucoma medications, cataract surgery, the number of previous glaucoma surgeries, the needling procedure and possibly diabetes) $[16,17]$ and conjunctival to scleral fibrogenesis is clearly indicated as the

Table 1 IOP (intra ocular pressure)

\begin{tabular}{|c|c|c|c|c|c|c|c|c|c|}
\hline \multirow[t]{2}{*}{ Patient } & \multicolumn{9}{|c|}{ IOP $(\mathrm{mmHg})$} \\
\hline & PRE-OP & Day 1 & At 1 week & At 2 weeks & At 1 month & At 3 months & At 9 months & At & follow-up \\
\hline 1 & 19 & 6 & 7 & 6 & 8 & 6 & 6 & 8 & $36 \mathrm{~m}$ \\
\hline 2 & 21 & 10 & 11 & 22 & 10 & 10 & 13 & 15 & $12 \mathrm{~m}$ \\
\hline 3 & 19 & 9 & 10 & 10 & 11 & 10 & 10 & 13 & $18 \mathrm{~m}$ \\
\hline $4^{*}$ & 22 & 10 & 10 & 17 & 9 & 12 & 14 & 15 & $20 \mathrm{~m}$ \\
\hline $5^{* *}$ & 20 & 9 & 10 & 10 & 10 & 11 & 11 & 14 & $17 \mathrm{~m}$ \\
\hline
\end{tabular}

$m$ months

*Subsequent needling, additional beta-blocker therapy

** Surgical failure history 


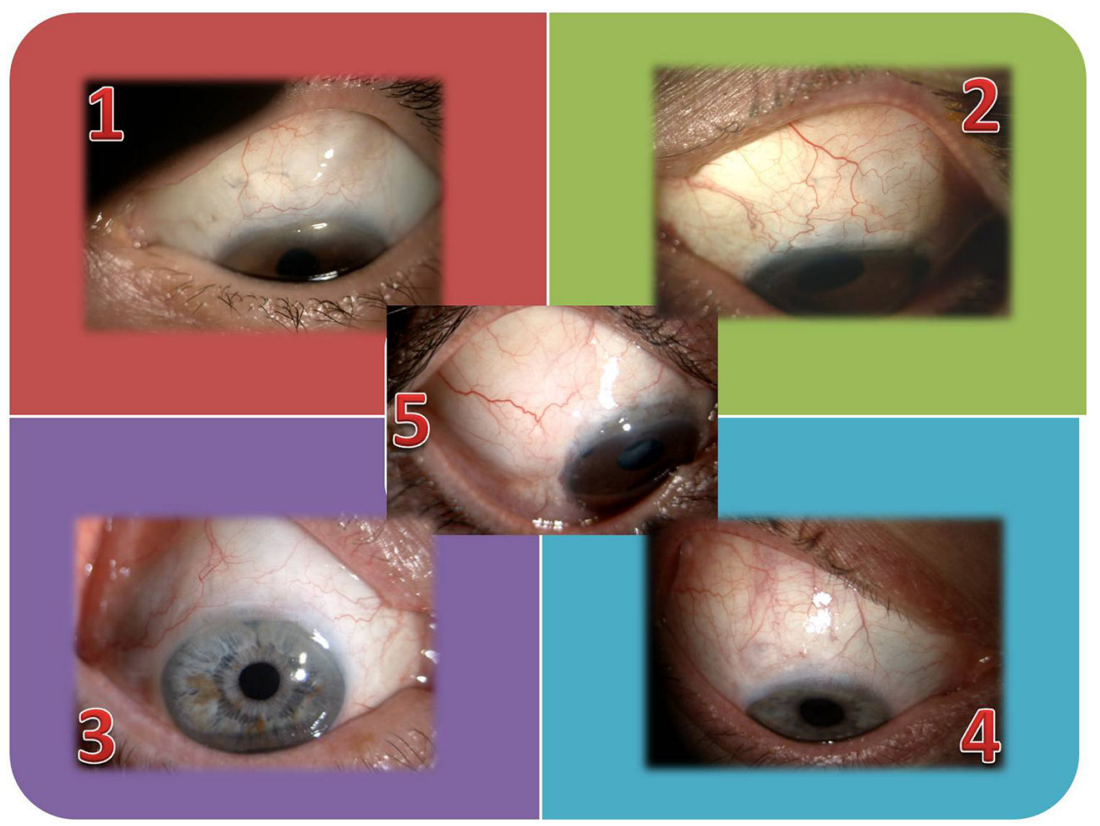

Fig. 1 Bleb photographs at 1 year of follow-up

main cause of glaucoma filtration surgery failure [18-20].

In response to surgical injury, recruitment of pro-inflammatory and pro-fibrogenic cytokines and chemokines into the aqueous leads to cellular migration and attraction of neutrophils [18]. Monocytes differentiate into tissue macrophages with critical effects on regulation of healing process through several growth factors; among these, the platelet-derived growth factor (PDGF) and the transforming growth factorbeta (TGF- $\beta$ ) are the most important pro-fibrogenic cytokines implicated in the activation of Tenon's fibroblasts together with increased extracellular matrix component synthesis. TGF$\beta$ directly induces the differentiation of fibroblasts into collagen-secreting myofibroblasts [19] and fibrillin-1 and fibronectin deposition on bleb site [20] representing the most potent stimulating factor for type I collagen gene transcription [21]. We hypothesize that the satisfactory clinical course after surgery remarkably observed in our patients series, may be related to a possible modulatory role of $\mathrm{CY}$ on the fibrosis cascade.

In MS immunologic milieu CY shows multiple selective effects on the immune system (Fig. 1). In particular it enhances $\mathrm{T}_{\mathrm{H}} 3_{\text {reg }}$ cell response [14], a unique T-cell subset primarily secreting transforming growth factor (TGF)-beta and IL-10 [23]: while macrophage-derived TGF$\beta$ generally shows wound-healing and pro-fibrotic activity, TGF- $\beta$ secreted from adaptive/ induced $\mathrm{T}_{\mathrm{H}} 3_{\text {reg }}$ cells shows immunosuppressive effects [24]. Furthermore the function of IL-10 as anti-fibrotic cytokine $[25,26]$, as well as its role in inhibition of collagen synthesis of the macrophages [27] are reported in several studies.

With regard to fibrosis process control, an interesting study in patients suffering from systemic sclerosis, reported for the first time CY effect on reducing fibrillin ECM deposition and limiting matrix remodeling [28]. The effectiveness on progressive fibrosis and the safety of low intravenous doses of CY was also reported in severe ocular cicatricial pemphigoid [29].

Moreover, angiogenesis that typically begins soon after wound formation, may contribute to tissue fibrosis [4]. Pro-angiogenic factors, such as fibroblast growth factor (FGF) and vascular endothelial growth factor (VEGF) are released from platelets and macrophages [22]. Intriguingly, several oncological studies [30] have shown an anti-angiogenic effect of frequent, even daily minimally-toxic dose administration 
of CY [31], regardless of tumor microenvironment [32]. The above-mentionated studies support the interpretation that CY pharmacological effects may interfere with scarring issues of the bleb in our patients, drastically slowing down the reparative process that leads to surgical failure; according to the literature this observation was never reported, probably due to sporadic cases observed in smaller center. We are aware that because of the limited number of cases and the absence of a comparative study design, we can not be sure that the surgical success is only due to CY. It would be of interest to confirm these observations by future controlled clinical studies. Moreover, pathology studies aimed at analyzing the conjunctival and scleral reparative process in such patients, would provide precious information regarding anti-fibrotic effect of CY not only of ophthalmologic interest but possibly useful to other fields of medicine.

\section{ACKNOWLEDGEMENTS}

The authors thank the participants of the study.

Funding. No funding or sponsorship was received for this study or publication of this article. The article processing charges were funded by the authors.

Authorship. All named authors meet the International Committee of Medical Journal Editors criteria (ICMJE) for authorship for this article, take responsibility for the integrity of the work as a whole, and have given their approval for this version to be published.

Disclosures. Andrea Giudiceandrea, Maria Emanuela Toro, Andrea Scupola, Aldo Caporossi, Viviana Nociti, Massimiliano Mirabella and Tommaso Salgarello have nothing to disclose.

Compliance with ethics guidelines. Informed consent was obtained from all individual patients for being included in the series.

Data availability. Data generate during this study are partially included in this published article; all data are available from the corresponding author on reasonable request.

Open Access. This article is distributed under the terms of the Creative Commons Attribution-NonCommercial 4.0 International License (http://creativecommons.org/licenses/ by-nc/4.0/), which permits any noncommercial use, distribution, and reproduction in any medium, provided you give appropriate credit to the original author(s) and the source, provide a link to the Creative Commons license, and indicate if changes were made.

\section{REFERENCES}

1. Burr J, Azuara-Blanco A, Avenell A. Medical versus surgical interventions for open angle glaucoma. Cochrane Database Syst Rev. 2005;2:CD004399.

2. Coleman AL. Advances in glaucoma treatment and management: surgery. Invest Ophthalmol Vis Sci. 2012;53:2491-4.

3. Jampel HD, McGuigan LJ, Dunkelberger GR, L'Hernault NL, Quigley HA. Cellular proliferation after experimental glaucoma filtration surgery. Arch Ophthalmol. 1988;106:89-94.

4. Yamanaka O, Kitano-Izutani A, Tomoyose K, Reinach PS. Pathobiology of wound healing after glaucoma filtration surgery. BMC Ophthalmol. 2015;15(Suppl 1):157.

5. Georgoulas S, Dahlmann-Noor A, Brocchini S, Khaw PT. Modulation of wound healing during and after glaucoma surgery. Prog Brain Res. 2008;173:237-54.

6. Razeghinejad MR, Fudemberg SJ, Spaeth GL. The changing conceptual basis of trabeculectomy: a review of past and current surgical techniques. Surv Ophthalmol. 2012;57(1):1-25.

7. Hafler DA, Slavik JM, Anderson DE, O'Connor KC, De Jager P, Baecher-Allan C. Multiple sclerosis. Immunol Rev. 2005;204:208-31.

8. Emadi A, Jones RJ, Brodsky RA. Cyclophosphamide and cancer: golden anniversary. Nat Rev Clin Oncol. 2009;6:638-47.

9. Sistigu A, Viaud S, Chaput N, Bracci L, Proietti E, et al. Immunomodulatory effects of cyclophosphamide and implementations for vaccine design. Semin Immunopathol. 2011;33:369-83. 
10. Mauch E, Kornhuber HH, Pfrommer U, Hähnel A, Laufen H, Krapf H. Effective treatment of chronically progressive multiple sclerosis with low-dose cyclophosphamide with minor side-effects. Eur Arch Psychiatry Neurol Sci. 1989;238(3):115-7.

11. Rinaldi L, Perini P, Calabrese M, Gallo P. Cyclophosphamide as second-line therapy in multiple sclerosis: benefits and risks. Neurol Sci. 2009;30(Suppl 2):S171-3.

12. Hafler DA, Orav J, Gertz R, Stazzone L, Weiner HL. Immunologiceffects of cyclophosphamide/ACTH in patients with chronic progressive multiple sclerosis. J Neuroimmunol. 1991;32:149-58.

13. Takashima H, Smith DR, Fukaura H, et al. Pulse cyclophosphamide plus methylprednisolone induces myelin-antigen-specific IL-4-secreting T cells in multiple sclerosis patients. Clin Immunol Immunopathol. 1998;88:28-34.

14. Weiner HL, Cohen JA. Treatment of multiple sclerosis with cyclophosphamide: critical review of clinical and immunologic effects. Mult Scler. 2002;8(2):142-54.

15. The AGIS investigators. The advanced glaucoma intervention study (AGIS): 7 . the relationship between control of intraocular pressure and visual field deterioration. Am J Ophthalmol. 2000;130(4):429-40.

16. Casson R, Rahman R, Salmon JF. Long term results and complications of trabeculectomy augmented with low dose mitomycin $\mathrm{C}$ in patients at risk for filtration failure. Br J Ophthalmol. 2001;85:686-8.

17. Landers J, Martin K, Sarkies N, Bourne R, Watson P. A twenty-year follow-up study of trabeculectomy: risk factors and outcomes. Ophthalmology. 2012;119(4):694-702.

18. Lama PJ, Fechtner RD. Antifibrotics and wound healing in glaucoma surgery. Surv Ophthalmol. 2003;48(3):314-46.

19. Milani BY, Milani FY, Park D-w, Namavari A, Shah J, Amirjamshidi H, Ying H, Djalilian AR. Rapamycin inhibits the production of myofibroblasts and reduces corneal scarring after photorefractive keratectomy. Invest Ophthalmol Vis Sci. 2013; 54(12):7424-30.

20. Saika S, Yamanaka O, Baba Y, Kawashima Y, Shirai K, Miyamoto T, Okada Y, Ohnishi Y, Ooshima A. Accumulation of latent transforming growth factorbeta binding protein-1 and TGF beta 1 in extracellular matrix of filtering bleb and of cultured human subconjunctival fibroblasts. Accumulation of latent transforming growth factor-beta binding protein-1 and TGF beta 1 in extracellular matrix of filtering bleb and of cultured human subconjunctival fibroblasts. Graefe's Arch Clin Exp Ophthalmol. 2001;239:234-241.

21. Ghosh AK, Quaggin SE, Vaughan DE. Molecular basis of organ fibrosis: potential therapeutic approaches. Exp Biol Med. 2013;238:461-81.

22. Chang L, Crowston JG, Cordeiro MF, Akbar AN, Khaw PT. The role of the immune system in conjunctival wound healing after glaucoma surgery. Surv Ophthalmol. 2000;45(1):49-68.

23. Jonuleit H, Schmitt E. ${ }^{\dagger}$ The Regulatory T cell family: distinct subsets and their interrelations. J Immunol. 2003;171(12):6323-7.

24. Peterson RA. Regulatory T-cells: diverse phenotypes integral to immune homeostasis and suppression. Toxicol Pathol. 2012;40(2):186-204.

25. Jin Y, Liu R, Xie J, Xiong H, He JC, Chen N. IInterleukin-10 deficiency aggravates kidney inflammation and fibrosis in the unilateral ureteral obstruction mouse model. Lab Invest. 2013;93(7):801-11.

26. Sziksz E, Pap D, Lippai R, Béres NJ, Fekete A, Szabó AJ, Vannay Á. Fibrosis related inflammatory mediators: role of the IL-10 cytokine family mediators of inflammation. 2015;Article ID 764641:15.

27. Mrowietz U, Seifert O. Keloid scarring: new treatments ahead. Actas Dermo-Sifiliográficas. 2009;100(Supplement 2):75-83.

28. Villano, et al. Systemic sclerosis sera affect fibrillin-1 deposition by dermal blood microvascular endothelial cells: therapeutic implications of cyclophosphamide. Arthritis Res Ther. 2013;15:R90.

29. Friedman J, Marcovich AL, Kleinmann G, Schattner A. Low-dose pulsed intravenous cyclophosphamide for severe ocular cicatricial pemphigoid in elderly patients. Cornea. 2014;33(10):1066-70.

30. Kerbel RS, Kamen BA. The anti-angiogenic basis of metronomic chemotherapy. Nat Rev Cancer. 2004;4(6):423-36.

31. Drevs J, Fakler J, Eisele S, Medinger M, Bing G, Esser $\mathrm{N}$, Marmé D, Unger C. Antiangiogenic potency of various chemotherapeutic drugs for metronomic chemotherapy. Anticancer Res. 2004;24(3a):1759-63.

32. Browder T, Butterfield CE, Kräling BM, Shi B, Marshall B, O'Reilly MS, Folkman J. Antiangiogenic scheduling of chemotherapy improves efficacy against experimental drug-resistant cancer. J Cancer Res. 2000;60(7):1878-86. 